\title{
Tree Composition and Ecological Structure of Akak Forest Area
}

\author{
Agbor James Ayamba ${ }^{1,2}$, Nkwatoh Athanasius Fuashi ${ }^{1}, \&$ Ayuk Elizabeth Orock ${ }^{1}$ \\ ${ }^{1}$ Department of Environmental Science, University of Buea, Cameroon \\ ${ }^{2}$ Ajemalebu Self Help, Kumba, South West Region, Cameroon \\ Correspondence: Agbor James Ayamba, Department of Environmental Science, University of Buea, Cameroon. \\ Tel: 237-652-079-481. E-mail: ayamba87@yahoo.com
}

Received: August 2, 2019

Accepted: September 11, 2019

Online Published: October 12, 2019

doi:10.5539/enrr.v9n4p23

URL: https://doi.org/10.5539/enrr.v9n4p23

\begin{abstract}
Tree composition and ecological structure were assessed in Akak forest area with the objective of assessing the floristic composition and the regeneration potentials. The study was carried out between April 2018 to February 2019. A total of 49 logged stumps were selected within the Akak forest spanning a period of 5 years and $20 \mathrm{~m} \mathrm{x}$ $20 \mathrm{~m}$ transects were demarcated. All plants species $<1 \mathrm{~cm}$ and above were identified and recorded. Results revealed that a total of 5239 individuals from 71 families, 216 genera and 384species were identified in the study area. The maximum plants species was recorded in the year 2015 (376 species). The maximum number of species and regeneration potentials was found in the family Fabaceae, (99 species) and (31) respectively. Baphia nitida, Musanga cecropioides and Angylocalyx pynaertii were the most dominant plants specie in the years 2013, 2015 and 2017 respectively. The year 2017 depicts the highest Simpson diversity with value of $(0.989)$ while the year 2015 show the highest Simpson dominance with value of (0.013). The year 2013 show a highest Shannon evenness with value of (0.4879). Logged compartment 2015 has a highest fisher alpha with value of 137.7 depicting highest specie richness The Shortest Euclidean distance of 123.44 between year 2013 and 2017 show that they both have many plants species that are similar. Evidently the forest area is very rich in trees in the lower diameter classes, and the structure of the Akak forest area is J reverse indicating that the forest is growing to climax.
\end{abstract}

Keywords: Floristic Composition, Regeneration Potentials, Species Diversity, Species Similarities

\section{Introduction}

The sustainable management of the natural resources of the various world ecosystems has been a global concern for many centuries, and in 1990 was stated as an important point in the United Nations' millennium development goals (United Nation [UN],1990). Two years later, the Agenda 21 of the Rio summit placed a specific emphasis on the sustainable management of forest resources worldwide (UN, 1992), because forests constitute the planet's largest terrestrial ecosystems and the richest source of biological diversity. Included amongst these valuable forest ecosystems, the management of which has been a cause of much concern, are the tropical rainforests.

The tropical rainforest has been identified as the most biologically diverse terrestrial ecosystem on earth (Turner, 2001; Gillespie, Brock, \& Wright., 2004; Onyekwelu, Mosandl, \& Stimm, 2008; Schmitt et al., 2009; FAO, 2010; IUCN, 2010). In terms of tree composition and species diversity, tropical rain forests are Earth"s most complex ecosystems (Gebreselasse, 2011). Trees are often the most conspicuous plant life form in a typical tropical rainforest. The rainforest act as main repository of the genetic diversity of both flora and fauna.

Natural regeneration of the plant species of a forest is essential for conservation and maintenance of biodiversity (Hossain, Rahman, Hoque, \& Khairul, 2004). It helps in the development of plant population of an area over time and space. This is a complex ecological process which involves dispersal of propagating materials, reproduction and establishment of seedlings in relation to environmental factors (Barnes, Zak, Denton, \& Spurr, 1998).

Sustainable management of natural forests depends on their ability to regenerate. In this respect, understanding natural regeneration processes and the distribution of recruits is of paramount importance to estimating the future forest structure and composition (Tesfaye, Teketay, \& Fetene, 2002; Ceccon, Huante, \& Rincon, 2006) and to create or enforce conservation regulations (Schaafsma et al., 2011).

The regeneration status/potential of a species can be assessed from the population dynamics of seedlings and saplings in the forest community (Duchok, Kenyusen, Ashalata, Ashish, \& Khan 2005). Several studies have 
predicted the regeneration status of tree species based on the age and diameter structure of their populations (Pritts \& Hancock, 1983; Bhuyan, Khan, \& Tripathi 2003). A population structure characterized by the presence of sufficient number of seedlings, saplings and young trees exhibits satisfactory regeneration potential, while an inadequate number of seedlings and saplings is indicative of poor regeneration potential (Saxena \& Singh, 1984).

Cameroon is the most biologically rich country known to date on the African continent (Sunderland, Comiskey, Besong, Mboh, Fonwebon, \& Dione, 2003). It encompasses an intricate mosaic of diverse habitats with moist tropical forest dominating the south and south-east and covering 54\% of the country, mountain forest and savannah in the highlands and sub-Sahelian savannah and near desert in the far north (Sunderland et al., 2003).

These diverse habitats harbour more than 9,000 species of plants, 160 species of which are endemic (Fonge, Tchetcha, \& Nkembi, 2013). The majority of the endemic taxa are concentrated around Mount Cameroon and other highland areas (Fonge et al., 2013). During the last few decades, deforestation of tropical forests areas has accelerated at an alarming rate as extensive areas of forest are being cleared every year (Tchouto, 2004). Man affects the forest ecosystem with activities such as agro industries, shifting cultivation, and hunting. There has been an overwhelming concern about the loss of tropical diversity and an emphasis on the identification of biodiversity hot spots in an attempt to optimize conservation strategies (Beentje, 1996).

Several studies indicate that logging directly affects species composition and structure of the forests ecosystem (Silva, De Carvalho, De Lopes, De Almeida, Costa, De Oliveira, Vanclay, \& Skovsgaard, 1995; Hall, Harris, Medjibe, \& Ashton, 2003; Okuda, Suzuki, Adachi, Quah, Hussein, \& Manokaran, 2003; Asase, Asiatokor, \& Ofori-Frimpong, 2014). Preserving forest biodiversity without harming economic interests is a big challenge for nations with forests. Local biodiversity loss due to timber extraction activities can disrupt the long-term resilience of forests, which may in turn cascade into an impoverished delivery of ecosystems services, ultimately affecting also human well-being.

Extracting timber or other products changes the tree age structure, composition of tree species and vertical stratification, thereby affecting local temperature, light, moisture, soil, and litter conditions. This results in changes or complete removal of microhabitats (such as dead wood, cavities, root plates or mature trees) that host forest biodiversity. The magnitude of impact on species diversity increases as the forest ecosystem has been under serious pressure from farmland extension (Akinsanmi, 1999).

These changes have caused the loss of some plant species and a decline in the biodiversity conservation status of the Akak forest area. The sustainable management and use of these resources is essential for the nation's economic and environmental security (Akinsanmi, 1999). More over, the Akak forest area serve as buffer zone for most wildlife species moving between Korup region (Korup National Park, Nta ali, Rumpi hills and Ejagham forest reserves). The ecosystem is a centre of high endemism for many taxa (plants, amphibians, mammals, and birds), and its destruction could lead to the local extinction of globally threatened biodiversity (plants, mammals etc.), watershed destruction, and degradation of livelihood systems, property, and lives. conservation.

Diversity studies carried out in Cameroon have covered many parts of the country but left out certain regions despite their richness in plant diversity (Mbolo, 2002). Akak forest area in part of these uncovered areas were there have been relatively few studies on the tree specie composition and diversity. There is a need to provide adequate quantitative and qualitative ecological data to guide forest owners and managers in fashioning out realistic and effective management strategies.

The objectives of this study were to identify tree composition and ecological structure of Akak forest area, specifically: (i) to access the floristic composition (ii) to know the regeneration potentials; and (iii) examine the ecological structure of the Akak forest area. This study is intended to provide baseline information on tree specie composition and diversity in the Akak forest area of Cameroon.

\section{Materials and Methods}

\subsection{Location of the Study Area}

The Akak forest area of Cameroon is located between $5^{\circ} 20^{\prime}-5^{\circ} 25^{\prime} \mathrm{N}$ latitude and $9^{\circ} 12^{\prime}-9^{\circ} 30^{\prime}$ E longitude (Figure 1). Akak is comprised of semi-deciduous lowland rainforest of the Guineo-Congolian type (Kenfack Thomas, Chuyong, \& Condit, 2007). Precipitation is unimodal, with an annual average around $4100 \mathrm{~mm}$ (Nchanji \& Plumptre, 2003), with a three-month dry season from December to February (Groenendijk, 2015). The topography is relatively flat. Human interventions, primarily establishing large plantations of cash crops (palm oil, coffee), as well as natural factors, such as elephant disturbance and windfalls, have created large gaps in these forests. Logged forest sites are located in the "heart" of the MPL (Mukete Plantations Limited) concession and the forests of this area have undergone logging, both formal and informal, from 1995 to the present. 


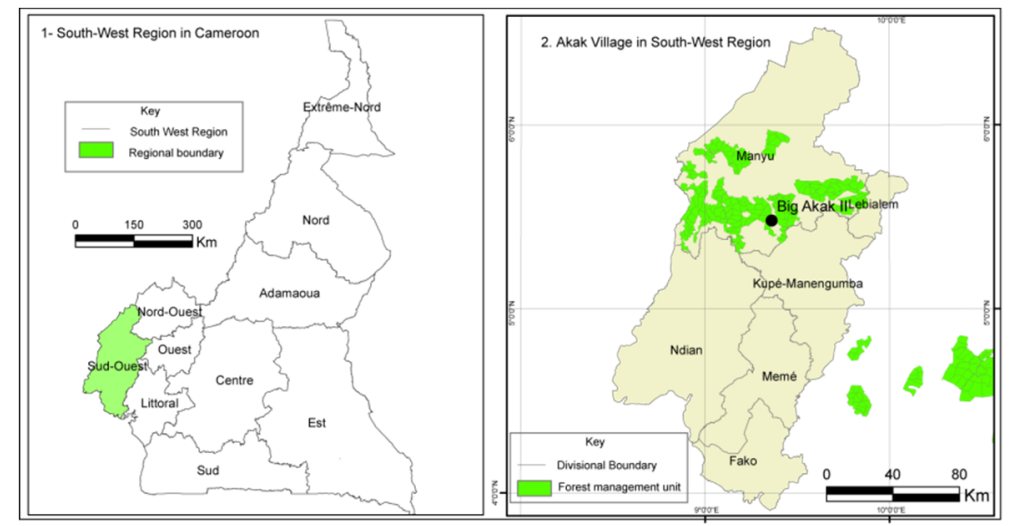

Figure 1. Akak forest area. Source: Adapted from the Cameroon Atlas 2019

\subsection{Method}

\subsubsection{Sampling Plots Establishment for Floristic Survey}

A total of 49 logged stumps were selected within the Akak forest spanning a period of 5 years. All three forest compartments had been selectively logged in different year: in 2013, 2015 and 2017. Within each forest compartment, logged stumps were selected randomly, at each stump, a $20 \times 20 \mathrm{~m}$ transects was demarcated along a disturbance gradient (Figure 2). The transects were demarcated in such manner that the logged stumps will be in the middle and one side of the transects were the highly disturbed side where the tree fell and the other sides was the sides opposite the felling direction, which was least disturbed (Figure 3). A total of 49 transects was created.

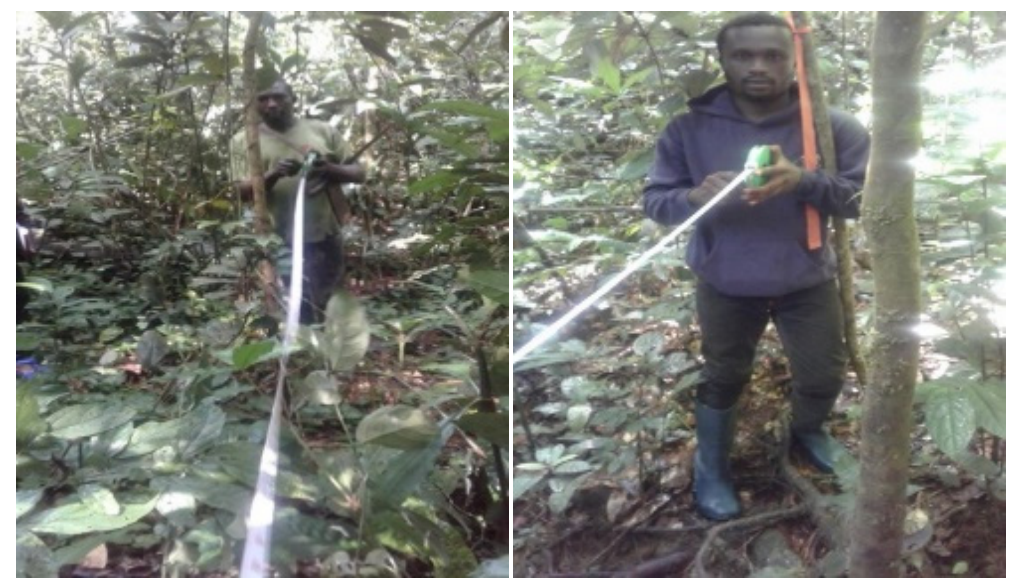

Figure 2. Demarcation of transects, Source field work, 2018

Table 1. Sampling transects

\begin{tabular}{ll}
\hline Year of logging & Logged stumps \\
\hline 2013 & 14 \\
2015 & 20 \\
2017 & 15 \\
Total & $\mathbf{4 9}$ \\
\hline
\end{tabular}




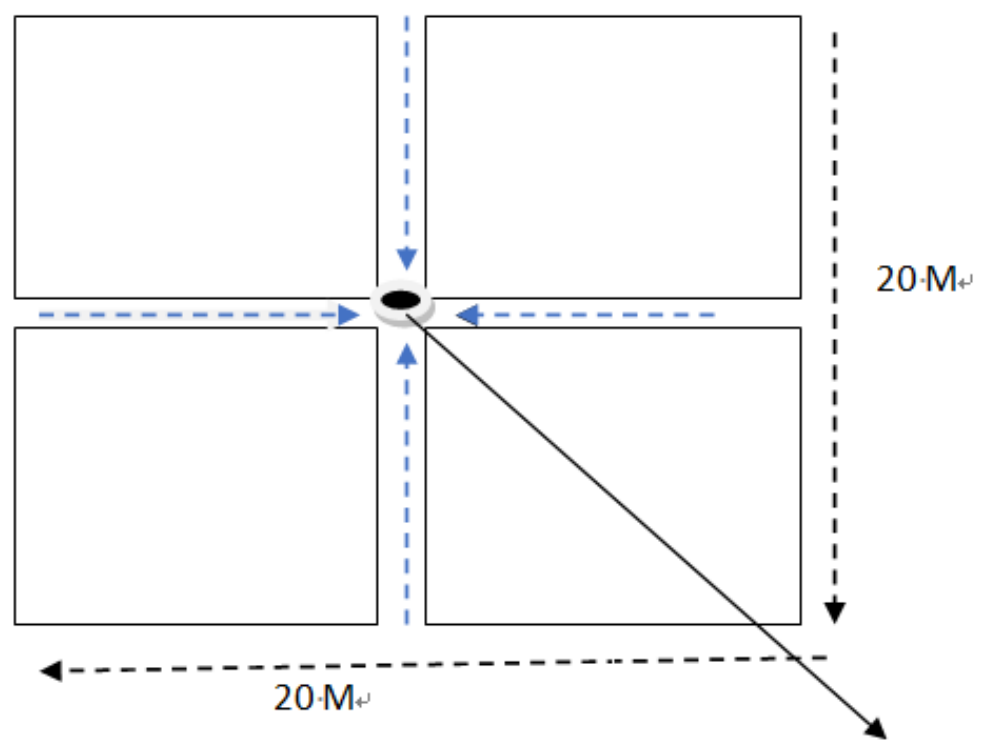

STUMP

Figure 3. Sampling transects design

\subsubsection{Data Collection}

All plants taller $<2 \mathrm{~cm}$ were identified to the species level and counted within the $20 \mathrm{~m} \times 20 \mathrm{~m}$ transect. The inventory included tree seedlings were those with diameter size class $<2 \mathrm{~cm}$ as recommended by Luoga and Lejju (2004) saplings were the young trees with diameter size class of $(2-6 \mathrm{~cm})$ small trees $(>6-9.9 \mathrm{~cm})$, medium-sized trees $(10-29.9 \mathrm{~cm})$, and large trees $(>29.9 \mathrm{~cm})$ following a grouping done by Kenfack et al. (2007). The diameter (DBH) of target individuals was recorded at $1.3 \mathrm{~m}$ stem height, or at $50 \mathrm{~cm}$ above buttresses where these were present at $1.3 \mathrm{~m}$ stem height.

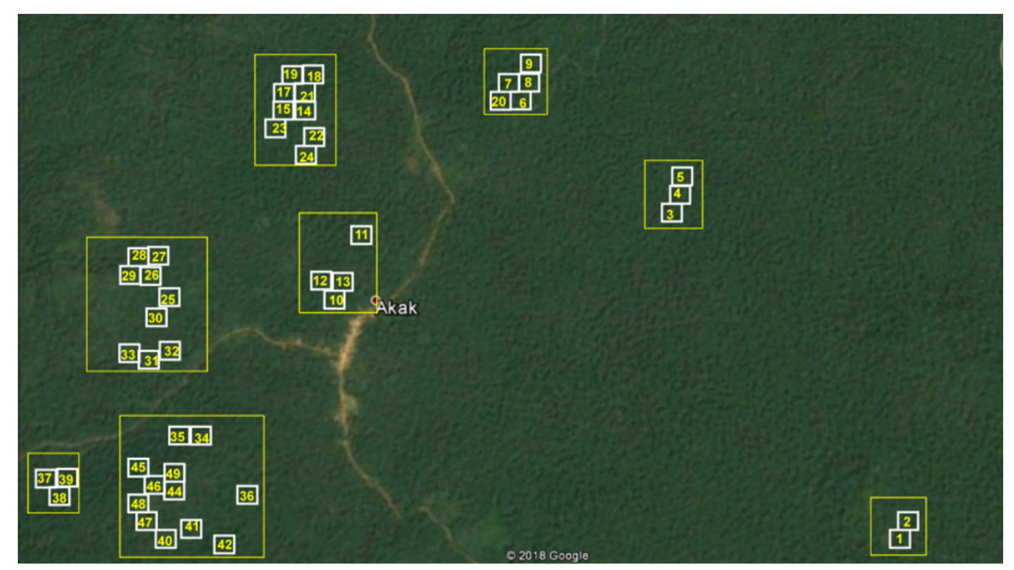

Figure 4. The distribution of sampling transects, source field work, 2018

\subsubsection{Data Analysis}

Community structure was analyzed through various diversity indices (Shannon-Weaver eveness Indices, Simpson's Indices of diversity and dominance and Fishers' Alpha diversity Index) according to the following formulae:

Data for abundance of species in the transect was first tested for normality, and due to a negative normality test $(\mathrm{p}>0.05)$ the plots were compared using Kruskal-Wallis non parametric analysis of variance. Following this, the community structure was analyzed through various diversity indices (Shannon-Weaver Evenness Indices, 
Simpson's Indices of diversity and dominance and Fishers' Alpha Diversity Index) according to the following formulae:

\section{Shannon Index of evenness}

Shannon evenness' was calculated as follows:

$$
\text { Shannon } H=\sum_{i}^{i=1} \text { piLnpi }
$$

Where $\mathrm{H}=$ the Shannon Index of diversity, $\mathrm{pi}=$ relative species abundance, $\ln =$ natural logarithm.

\section{Species Evenness:}

$$
\text { Evenness }=\frac{H}{H \max }
$$

Where Hmax is the maximum possible diversity in the ecosystem.

\section{Simpsons Indices of diversity:}

\section{Simpsons Index of dominance:}

$$
D=\sum_{i}^{i=1} p i^{2}
$$

Where $\mathrm{pi}=$ relative abundance of species.

\section{Simpsons Index of diversity:}

$$
\mathbf{1}-\boldsymbol{D}=\left(1-\sum_{i}^{i=1} p i^{2}\right)
$$

The value of this index ranges between 0 and 1; the greater the value, the greater the sample diversity. The index represents the probability that two individuals randomly selected from a sample will belong to different species.

\section{Simpsons reciprocal Index}

$$
\mathbf{1} / \boldsymbol{D}=1 /\left(\sum_{i}^{i=1} p i^{2}\right)
$$

The value of this index starts with 1 as the lowest possible figure. The higher the value, the greater the diversity. The maximum value is the number of species in the sample.

\section{Fishers Alpha}

$$
\text { Fishers Alpha }=a x, \frac{(a x)^{r}}{r}, \frac{(a x)^{r 2}}{r^{2}}, \ldots, \frac{(a x)^{n}}{n}, \ldots
$$

Importance value Indices of the different species were calculated as follows:

IVI = Relative Dominance + Relative Density + Relative Frequency

Where:

-Relative dominance $=$ Basal Area of each species $/$ Total Basal area of the site;

-Relative density $=$ Abundance of each species/ Total number of individuals at the site;

-Relative Frequency = Frequency of occurrence of each species across the subplots/Total Frequency at the site.

Data on species abundance was plotted across diameter classes to determine the state of the forest (whether the forest is growing of mature and declining).

All these analyses were done in the Minitab Version 17 Statistical Package (Minitab Inc., PA, USA) at $\alpha=0.05$. Similarities of species were calculated using Euclidean and correlation distance.

\section{Results}

\subsection{Floristic Composition of Akak Forest Area}

A total of 5239 individuals from 71 families, 216 genera and 384species were identified in the study area (2013, 2015 and 2017) (Table 2). 1249 individuals were seedlings $<2 \mathrm{~cm}$ tall, and 1458 individuals were saplings $2-6 \mathrm{~cm}$ tall, 156 individuals were small trees of $>6-9.99 \mathrm{~cm}, 1244$ individuals were medium size trees $10-29.99 \mathrm{~cm}$ and 918 individuals were large trees $>29.99 \mathrm{~cm}$. The individuals in the different years do not differ significantly $(P<0.05)$ (Table 3). 
Table 2. Comparison of 0.5 ha sample size for the different years $(2013,2015$ and 2017)

\begin{tabular}{llll}
\hline Variable & 2013 & 2015 & 2017 \\
\hline Number of species & 308 & 376 & 351 \\
Number of families & 63 & 65 & 56 \\
Number of genera & 156 & 183 & 164 \\
Individuals & 1449 & 1826 & 1964 \\
Basal area/0.5 ha & 0.28 & 0.27 & 0.32 \\
Density/0.5 ha & 1449 & 1826 & 1964 \\
\hline
\end{tabular}

The results revealed that the maximum plants species was recorded in 2015 (376 species), followed by 351 species in 2017 and 308 species in 2013. (Table 4). The plant species in the different years do not differ significantly (Table 21). This means that the Akak forest area has similar plant species.

Table 3. Kruskal Wallis test on taxa and individual versus years

\begin{tabular}{|c|c|c|c|c|c|c|c|c|}
\hline Categories & Year & $\mathbf{N}$ & Median & Ave Ranking & $\mathbf{Z}$ & $\mathbf{H}$ & DF & $\mathbf{P}$ \\
\hline \multirow{4}{*}{ Taxa_S versus Year } & 2013 & 14 & 61.50 & 23.8 & -0.37 & & & \\
\hline & 2015 & 20 & 54.00 & 21.9 & -1.25 & 3.01 & 2 & 0.222 \\
\hline & 2017 & 15 & 69.0 & 30.2 & 1.69 & & & \\
\hline & Overall & 49 & & 25.0 & - & - & - & - \\
\hline \multirow{4}{*}{ Individuals versus Year } & 2013 & 14 & 107.50 & 23.0 & -0.62 & & & \\
\hline & 2015 & 20 & 94.50 & 22.4 & -1.05 & 2.99 & 2 & 0.225 \\
\hline & 2017 & 15 & 69.0 & 30.3 & 1.72 & & & \\
\hline & Overall & 49 & & 25.0 & - & - & - & - \\
\hline
\end{tabular}

Table 4. The distribution of families, genera and species in the Akak forest area

\begin{tabular}{|c|c|c|c|c|c|c|c|c|}
\hline & 2013 & & 2015 & & 2017 & & & \\
\hline FAMILY & GENERA & SPECIES & GENERA & SPECIES & GENERA & SPECIES & $\begin{array}{l}\text { Total } \\
\text { Genera }\end{array}$ & $\begin{array}{l}\text { Total } \\
\text { species }\end{array}$ \\
\hline Ancanthaceae & - & - & 1 & 3 & 1 & 1 & 2 & 4 \\
\hline Anacardiaceae & 3 & 8 & 4 & 12 & 2 & 9 & 8 & 13 \\
\hline Annonaceae & 9 & 15 & 11 & 16 & 10 & 20 & 30 & 51 \\
\hline Apocynaceae & 9 & 13 & 8 & 12 & 7 & 11 & 24 & 39 \\
\hline Araceae & 2 & 2 & 1 & 4 & 2 & 2 & 5 & 8 \\
\hline Arecaceae & 2 & 4 & 3 & 8 & 3 & 6 & 8 & 14 \\
\hline Asparagaceae & - & - & - & - & 1 & 1 & 1 & 2 \\
\hline Asteraceae & 1 & 1 & 1 & 1 & - & - & 2 & 3 \\
\hline Bignoniaceae & 1 & 1 & 1 & 1 & 2 & 2 & 4 & 7 \\
\hline Burseraceae & 4 & 7 & 5 & 6 & 4 & 6 & 13 & 22 \\
\hline Cecropiaceae & 2 & 2 & 2 & 4 & 2 & 2 & 6 & 10 \\
\hline Celastraceae & 1 & 2 & 1 & 5 & 1 & 3 & 3 & 5 \\
\hline Clusiaceae & 2 & 7 & 1 & 4 & 2 & 7 & 5 & 8 \\
\hline Combretaceae & 1 & 1 & 1 & 3 & 1 & 2 & 3 & 5 \\
\hline Commelinaceae & 2 & 3 & 3 & 3 & - & - & 5 & 8 \\
\hline Connaraceae & 1 & 1 & 1 & 3 & 2 & 3 & 4 & 7 \\
\hline Convolvulaceae & - & - & 1 & 1 & - & - & 1 & 2 \\
\hline Cucurbitaceae & - & - & 1 & 2 & - & - & 1 & 2 \\
\hline Dichapetalaceae & 2 & 6 & 2 & 7 & 2 & 6 & 6 & 10 \\
\hline Dilleriaceace & - & - & - & - & 1 & 1 & 1 & 2 \\
\hline Dioscoreaceae & 1 & 3 & 1 & 4 & 1 & 1 & 3 & 5 \\
\hline Ebenaceae & 1 & 10 & 1 & 12 & 1 & 14 & 3 & 5 \\
\hline Euphorbiaceae & 9 & 14 & 11 & 21 & 1 & 16 & 21 & 33 \\
\hline Fabaceae & 20 & 35 & 23 & 33 & 20 & 31 & 63 & 106 \\
\hline Gentianaceae & 1 & 1 & 2 & 3 & 1 & 1 & 4 & 7 \\
\hline
\end{tabular}




\begin{tabular}{|c|c|c|c|c|c|c|c|c|}
\hline \multirow[b]{2}{*}{ FAMILY } & \multicolumn{2}{|l|}{2013} & \multicolumn{2}{|l|}{2015} & \multicolumn{4}{|l|}{2017} \\
\hline & GENERA & SPECIES & GENERA & SPECIES & GENERA & SPECIES & $\begin{array}{l}\text { Total } \\
\text { Genera }\end{array}$ & $\begin{array}{l}\text { Total } \\
\text { species }\end{array}$ \\
\hline Gnetaceae & 1 & 1 & 1 & 1 & - & - & 2 & 3 \\
\hline Huaceace & - & - & 1 & 1 & 1 & 1 & 2 & 4 \\
\hline Humiriaceace & - & - & - & - & 1 & 1 & 1 & 2 \\
\hline Icacinaceae & 1 & 6 & 2 & 4 & 2 & 2 & 5 & 9 \\
\hline Irvingiaceae & 2 & 2 & 3 & 4 & 2 & 4 & 7 & 12 \\
\hline Lamiaceae & 1 & 1 & 1 & 4 & 1 & 2 & 3 & 5 \\
\hline Lauraceae & 2 & 5 & 2 & 3 & 3 & 5 & 7 & 12 \\
\hline Lecythidaceae & 1 & 2 & 2 & 5 & 2 & 2 & 5 & 9 \\
\hline Leguminosae & 1 & 1 & - & - & - & - & 1 & 1 \\
\hline Loganiaceae & 1 & 3 & 1 & 2 & 1 & 3 & 3 & 5 \\
\hline Malvaceae & 9 & 21 & 8 & 24 & 10 & 34 & 27 & 45 \\
\hline Marantaceae & 1 & 2 & 2 & 5 & 1 & 2 & 4 & 7 \\
\hline Melastomataceae & - & - & 3 & 6 & 1 & 1 & 4 & 8 \\
\hline Meliaceae & 8 & 18 & 7 & 15 & 6 & 14 & 21 & 34 \\
\hline Memecylaceae & - & - & 1 & 1 & - & - & 1 & 2 \\
\hline Menispermaceae & 1 & 1 & 1 & 1 & 1 & 1 & 3 & 5 \\
\hline Monimiaceae & 1 & 1 & 1 & 1 & - & - & 2 & 3 \\
\hline Moraceae & 6 & 7 & 5 & 11 & 4 & 8 & 15 & 24 \\
\hline Myristicaceae & 4 & 6 & 1 & 2 & 3 & 3 & 8 & 12 \\
\hline Myrtaceae & 1 & 2 & 2 & 3 & 1 & 2 & 4 & 7 \\
\hline Ochnaceae & 4 & 7 & 2 & 2 & 3 & 3 & 9 & 14 \\
\hline Octoknemaceae & 1 & 1 & 1 & 1 & 1 & 2 & 3 & 5 \\
\hline Olacaceae & 4 & 7 & 4 & 8 & 4 & 11 & 12 & 20 \\
\hline Orchidaceae & - & - & 1 & 1 & - & - & 1 & 2 \\
\hline Pandaceae & 1 & 1 & 1 & 1 & 1 & 1 & 3 & 5 \\
\hline Passifloraceae & - & - & 1 & 1 & - & - & 1 & 2 \\
\hline Phyllanthaceae & 1 & 2 & 4 & 8 & 4 & 7 & 9 & 17 \\
\hline Piperaceae & - & - & 1 & 1 & - & - & 1 & 2 \\
\hline Polygalaceae & 1 & 2 & 1 & 1 & - & - & 2 & 3 \\
\hline Putranjivaceae & 1 & 10 & 1 & 12 & 1 & 11 & 3 & 5 \\
\hline Rhizophoraceae & - & - & - & - & 1 & 1 & 1 & 2 \\
\hline Rhamnaceae & 1 & 1 & 1 & 1 & - & - & 2 & 3 \\
\hline Rosaceae & 1 & 1 & 1 & 1 & 1 & 1 & 3 & 5 \\
\hline Rubiaceae & 11 & 29 & 15 & 35 & 13 & 27 & 39 & 67 \\
\hline Ruscaceae & 1 & 2 & 1 & 2 & 1 & 3 & 3 & 5 \\
\hline Rutaceae & 2 & 5 & 2 & 5 & 2 & 4 & 6 & 10 \\
\hline Solicaceae & - & - & 1 & 1 & - & - & 1 & 2 \\
\hline Sapindaceae & 2 & 2 & 2 & 3 & 3 & 6 & 7 & 12 \\
\hline Sapotaceae & 2 & 4 & 2 & 6 & 3 & 9 & 7 & 12 \\
\hline Tiliaceae & 1 & 1 & 1 & 1 & 1 & 1 & 3 & 5 \\
\hline Ulmaceae & 1 & 3 & 2 & 4 & 2 & 4 & 5 & 9 \\
\hline Urticaceae & 1 & 2 & 1 & 1 & - & - & 2 & 3 \\
\hline Verbenaceae & 1 & 2 & 1 & 1 & 1 & 4 & 3 & 5 \\
\hline Violaceae & 1 & 6 & 2 & 8 & 1 & 9 & 4 & 7 \\
\hline Vitaceae & 1 & 2 & & & 1 & 3 & 2 & 3 \\
\hline Zingiberaceae & 2 & 3 & 4 & 10 & 2 & 3 & 8 & 14 \\
\hline UK,Leguminaceace & - & - & - & - & 7 & 7 & 7 & 14 \\
\hline UK,Sapindaceace & - & - & - & - & 2 & 2 & 2 & 4 \\
\hline UK,Sapotaceace & - & - & - & - & 2 & 2 & 2 & 4 \\
\hline Total & 156 & 308 & 182 & 375 & 164 & 351 & - & - \\
\hline
\end{tabular}

*UK= Unknown 
The maximum number of species was found in the family Fabaceae (99 species) followed by Rubiaceae (91 species), Malvaceae (79 species), Annonaceae and Euphorbiaceae (51 species) each, and Meliaceae (47 species), Apocynaceae and Ebenaceae (36 species each), Putranjivaceae (33 species each) and rest 62 families comprising 28 to 1 species respectively (Table 22). The family Fabaceae for 2013 had the highest number of species (35) followed by 2015 (33 species) and 201731 (species), the family Rubiaceae for 2015 had the highest number of species (35) followed by 2013 (29 species) and 27 (species) 2017.

Table 5. Akak forest area population structure of tree individuals along girth class frequencies (DBH classes in (cm) / 0.5ha)

\begin{tabular}{|c|c|c|c|c|c|}
\hline $\begin{array}{l}\mathrm{DBH} \\
\text { classes }\end{array}$ & $\begin{array}{l}\text { Number of individual's } \\
\text { in } 2013\end{array}$ & $\begin{array}{l}\text { Number of } \\
\text { individual's in } \\
2015\end{array}$ & $\begin{array}{l}\text { Number of } \\
\text { individual's in } \\
2017\end{array}$ & Total & $\begin{array}{l}\text { Percentage of individual in the } \\
\text { Akak forest area }\end{array}$ \\
\hline$<10$ & 794 & 1078 & 1092 & 2964 & 56.5 \\
\hline $11-20$ & 239 & 285 & 338 & 862 & 16.4 \\
\hline 21-30 & 150 & 156 & 190 & 496 & 9.4 \\
\hline $31-40$ & 73 & 74 & 93 & 240 & 4.5 \\
\hline $41-50$ & 49 & 44 & 77 & 170 & 3.2 \\
\hline 51-60 & 25 & 41 & 36 & 102 & 1.9 \\
\hline $61-70$ & 22 & 47 & 34 & 103 & 1.9 \\
\hline $71-80$ & 14 & 22 & 15 & 51 & 0.9 \\
\hline $81-90$ & 6 & 16 & 23 & 45 & 0.8 \\
\hline 91-100 & 12 & 29 & 22 & 63 & 1.2 \\
\hline \multirow[t]{2}{*}{$>100$} & 65 & 34 & 44 & 143 & 2.7 \\
\hline & 1449 & 1826 & 1964 & 5239 & 100 \\
\hline
\end{tabular}

Population density of tree species across girth class interval in Akak forest area showed that around $56.5 \%$ of individuals belonged to $<10 \mathrm{~cm}$ DBH (Table 5). The highest number of species was also observed in the same category; the study area represents stands with good regeneration.

\subsection{Overall Importance of Plant Species in Akak Forest Area}

Table 6. Important value index (IVI) of plants species in 2013

\begin{tabular}{lllllll}
\hline Family & Genera & Scientific name & Frequency & Rel. Density & Rel. Dominance & IVI \\
\hline Fabaceae & Baphia & Baphia nitida & 13 & 3.588682 & 5.215358 & 21.80 \\
Ochnaceae & Lophira & Lophira alata & 6 & 1.449275 & 11.9965 & 19.45 \\
Fabaceae & Angylocalyx & Angylocalyx pynaertii & 11 & 5.383023 & 1.381469 & 17.76 \\
Malvaceae & Cola & Cola rostrata & 8 & 1.725328 & 6.672505 & 16.40 \\
Euphorbiaceae & Plagiostyles & Plagiostyles africana & 9 & 2.829538 & 4.50127 & 16.33 \\
Myristicaceae & Staudtia & Staudtia kamerunensis & 10 & 1.173223 & 2.955489 & 14.13 \\
Burseraceae & Pseudospondias & Pseudospondias sp.1 & 7 & 1.035197 & 5.205741 & 13.24 \\
Dichapetalaceae & Dichapetalum & Dichapetalum angolense & 9 & 1.311249 & 2.422935 & 12.73 \\
Ebenaceae & Diospyros & Diospyros sp.1 & 9 & 1.10421 & 2.510208 & 12.61 \\
Clusiaceae & Garcinia & Garcinia mannii & 9 & 1.794341 & 1.734889 & 12.53 \\
Fabaceae & Calpocalyx & Calpocalyx sp.1 & 8 & 2.00138 & 2.496233 & 12.50 \\
Malvaceae & Cola & Cola millenii & 11 & 1.449275 & 0.027519 & 12.48 \\
Rubiaceae & Tricalysia & Tricalysia sp.1 & 7 & 2.415459 & 2.486708 & 11.90 \\
Putranjivaceae & Drypetes & Drypetes sp.3 & 10 & 1.311249 & 0.077583 & 11.39 \\
Marantaceae & Halopegia & Halopegia sp.1 & 8 & 1.035197 & 1.990345 & 11.03 \\
Icacinaceae & Lasianthera & Lasianthera africana & 10 & 0.966184 & 0.026705 & 10.99 \\
Anacardiaceae & Sorindeia & Sorindeia macrophylla & 7 & 2.622498 & 1.358843 & 10.98 \\
Lecythidaceae & Napoleonaea & Napoleonaea sp.2 & 9 & 1.173223 & 0.001787 & 10.18 \\
Arecaceae & Elaeis & Elaeis guineensis & 1 & 0.414079 & 8.580546 & 9.99 \\
Annonaceae & Greenwayodendron & Greenwayodendron sp.1 & 6 & 0.89717 & 2.638314 & 9.54 \\
\hline
\end{tabular}


Top fifteen dominant tree species that are found in 2013 logged compartment in Akak forest area based on the Important Value Index (IVI) is shown in the (Table 6) The most dominant one is Baphia nitida followed by Lophira alata.

Table 7. Important value index of plants species in 2015

\begin{tabular}{lllllll}
\hline Family & Genera & Scientific name & Frequency & Rel. Density & Rel. Basal Area & IVI \\
\hline Cecropiaceae & Musanga & Musanga cecropioides & 12 & 6.626506024 & 7.084100042 & 25.71061 \\
Fabaceae & Angylocalyx & Angylocalyx pynaertii & 11 & 4.709748083 & 1.878068508 & 17.58782 \\
Euphorbiaceae & Macaranga & Macaranga sp.1 & 10 & 3.669222344 & 2.97462525 & 16.64385 \\
Moraceae & Treculia & Treculia obovoidea & 9 & 1.971522453 & 4.699689944 & 15.67121 \\
Fabaceae & Baphia & Baphia nitida & 11 & 1.807228916 & 0.671558059 & 13.47879 \\
Sapindaceae & Chytranthus & Chytranthus sp.1 & 11 & 1.040525739 & 0.702699968 & 12.74323 \\
Myristicaceae & Pycnanthus & Pycnanthus angolensis & 10 & 1.040525739 & 0.896888243 & 11.93741 \\
Ebenaceae & Diospyros & Diospyros suaveolens & 6 & 0.657174151 & 5.248817899 & 11.90599 \\
Burseraceae & Canarium & Canarium schweinfurthii & 9 & 1.588170865 & 0.947089694 & 11.53526 \\
Putranjivaceae & Drypetes & Drypetes sp.1 & 8 & 0.985761227 & 2.336333512 & 11.32209 \\
Malvaceae & Cola & Cola millenii & 9 & 1.588170865 & 0.675565761 & 11.26374 \\
Lecythidaceae & Napoleonaea & Napoleonaea sp.2 & 9 & 0.711938664 & 0.450184909 & 10.16212 \\
Rutaceae & Zanthoxylum & Zanthoxylum sp.1 & 7 & 1.150054765 & 1.957885512 & 10.10794 \\
Apocynaceae & Voacanga & Voacanga sp.1 & 8 & 1.369112815 & 0.557664961 & 9.926778 \\
Putranjivaceae & Drypetes & Drypetes molunduana & 8 & 1.423877327 & 0.38989974 & 9.813777 \\
Meliaceae & Trichilia & Trichilia sp.1 & 9 & 0.766703176 & 0.021563054 & 9.788266 \\
Anacardiaceae & Sorindeia & Sorindeia macrophylla & 8 & 1.204819277 & 0.259804833 & 9.464624 \\
Euphorbiaceae & Plagiostyles & Plagiostyles africana & 8 & 0.766703176 & 0.450138313 \\
Icacinaceae & Lasianthera & Lasianthera africana & 8 & 1.150054765 & 0.050213212 \\
Burseraceae & Santiria & Santiria tremari & 7 & 0.492880613 & 1.366860133 & 9.216841 \\
\hline & & & & 9.200268 \\
\hline
\end{tabular}

Top fifteen dominant tree species that are found in 2015 logged compartment in Akak forest area based on the Important Value Index (IVI) is shown in the (Table 7) The most dominant one is Musanga cecropioides followed by Angylocalyx pynaertii.

Table 8. Important value index of plants species in 2017

\begin{tabular}{|c|c|c|c|c|c|c|}
\hline Family & Genera & Scientific name & Frequency & Rel. Density & Rel. Basal Area & IVI \\
\hline Fabaceae & Angylocalyx & Angylocalyx pynaertii & 13 & 5.49898167 & 5.889832928 & 24.38881 \\
\hline Myristicaceae & Staudtia & Staudtia kamerunensis & 12 & 1.578411405 & 2.374044478 & 15.95246 \\
\hline Moraceae & Treculia & Treculia obovoidea & 11 & 2.545824847 & 2.370822708 & 15.91665 \\
\hline Malvaceae & Cola & Cola millenii & 12 & 1.985743381 & 0.570541027 & 14.55628 \\
\hline Anacardiaceae & Sorindeia & Sorindeia macrophylla & 10 & 2.036659878 & 2.379226046 & 14.41589 \\
\hline Violaceae & Rinorea & Rinorea dentata & 8 & 1.069246436 & 5.065841073 & 14.13509 \\
\hline Lecythidaceae & Napoleonaea & Napoleonaea sp.1 & 12 & 1.578411405 & 0.522226154 & 14.10064 \\
\hline Icacinaceae & Lasianthera & Lasianthera africana & 12 & 1.476578411 & 0.041221527 & 13.5178 \\
\hline Euphorbiaceae & Plagiostyles & Plagiostyles africana & 9 & 1.985743381 & 2.513852952 & 13.4996 \\
\hline Putranjivaceae & Drypetes & Drypetes molunduana & 10 & 2.138492872 & 1.325387039 & 13.46388 \\
\hline Dichapetalaceae & Tapura & Tapura africana & 10 & 1.731160896 & 0.794181287 & 12.52534 \\
\hline Fabaceae & Baphia & Baphia nitida & 8 & 0.916496945 & 3.459618809 & 12.37612 \\
\hline Icacinaceae & Lavigeria & Lavigeria sp.1 & 11 & 0.610997963 & 0.495053311 & 12.10605 \\
\hline Sapotaceae & Gambeya & Gambeya africanum & 8 & 0.712830957 & 2.737865704 & 11.4507 \\
\hline Rubiaceae & Corynanthe & Corynanthe sp.1 & 9 & 1.069246436 & 0.915642933 & 10.98489 \\
\hline Clusiaceae & Garcinia & Garcinia mannii & 9 & 1.018329939 & 0.900804273 & 10.91913 \\
\hline Fabaceae & Calpocalyx & Calpocalyx sp.1 & 8 & 1.018329939 & 1.732376611 & 10.75071 \\
\hline Lauraceae & Homalium & Homalium sp.1 & 9 & 0.967413442 & 0.683218623 & 10.65063 \\
\hline Huaceae & Afrostyrax & Afrostyrax lepidophyllus & 8 & 1.425661914 & 1.06909645 & 10.49476 \\
\hline
\end{tabular}




\begin{tabular}{llllll}
\hline Rutaceae & Zanthoxylum & Zanthoxylum sp.1 & 4 & 0.509164969 & 5.835968322
\end{tabular}

Top fifteen dominant tree species that are found in 2017 logged compartment in Akak forest area based on the Important Value Index (IVI) is shown in the (Table 8) The most dominant one is Angylocalyx pynaertii followed by Staudtia kamerunensis.

\subsection{Ecological Structure of the Akak Forest Area}

\subsubsection{Diversity}

Different biological indices, such as Shannon evenness $\left(\mathrm{e}^{\wedge} \mathrm{H} / \mathrm{S}\right)$ index's, Simpson's diversity index (1-D), Dominance (D) and Fisher_alpha, were evaluated for Akak forest area to reveal the natural regeneration status of recorded plant species (Table 9). The year 2017 depicts a higher diversity with value of (0.989) and least dominance with value of $(0.010)$ while 2015 show the least diverse with value of $(0.9869)$ and a higher dominance with value of (0.013), they differed significantly among the different years for both Dominance $(P=0.032)$ and diversity Simpson_1-D $(\mathrm{p}=0.033)$ (Table 10). The year 2013 show a higher evenness with value of $(0.4879)$ while the year 2015 show the lowest evenness with value of (0.4640) and did not differ significantly among the different years (Table 10). Logged compartment 2015 has a higher fisher alpha with value of 137.7 depicting higher specie richness while the year 2013 has the least specie richness with value of 120.9 .

Table 9. Comparison of 0.5ha sample size for the different years (2013, 2015 and 2017)

\begin{tabular}{llll}
\hline Variable & $\mathbf{2 0 1 3}$ & $\mathbf{2 0 1 5}$ & $\mathbf{2 0 1 7}$ \\
\hline Dominance_D & 0.012 & 0.013 & 0.010 \\
Simpson_1-D & 0.9879 & 0.9869 & 0.9892 \\
Evenness_e^H/S & 0.4908 & 0.4640 & 0.4817 \\
Fisher_alpha & 120.9 & 137.7 & 126.1 \\
\hline
\end{tabular}

Table 10. Kruskal-Wallis test on Dominance, Simpson and Evenness versus years

\begin{tabular}{|c|c|c|c|c|c|c|c|c|}
\hline Categories & Year & $\mathbf{N}$ & Median & Ave Ranking & $\mathbf{Z}$ & $\mathbf{H}$ & DF & $\mathbf{P}$ \\
\hline \multirow{4}{*}{ Dominance_D versus Year } & 2013 & 14 & 0.02999 & 29.0 & 1.24 & \multirow{4}{*}{6.91} & \multirow{3}{*}{2} & \multirow{3}{*}{0.032} \\
\hline & 2015 & 20 & 0.03145 & 28.3 & 1.32 & & & \\
\hline & 2017 & 15 & 0.02380 & 16.9 & 2.62 & & & \\
\hline & Overall & 49 & & 25.0 & & & \multirow{5}{*}{2} & \\
\hline \multirow{4}{*}{ Simpson_1-D versus Year } & 2013 & 14 & 0.9700 & 21.0 & -1.24 & \multirow{4}{*}{6.80} & & \multirow{4}{*}{0.033} \\
\hline & 2015 & 20 & 0.9686 & 21.8 & -1.30 & & & \\
\hline & 2017 & 15 & 0.9762 & 33.0 & 2.60 & & & \\
\hline & Overall & 49 & & 25.0 & & & & \\
\hline \multirow{4}{*}{ Evenness_e ${ }^{\wedge} \mathrm{H} / \mathrm{S}$ versus Year } & 2013 & 14 & 0.7652 & 23.3 & -0.53 & \multirow{4}{*}{0.35} & \multirow{4}{*}{2} & \multirow{4}{*}{0.837} \\
\hline & 2015 & 20 & 0.7979 & 26.3 & 0.51 & & & \\
\hline & 2017 & 15 & 0.7787 & 24.9 & -0.02 & & & \\
\hline & Overall & 49 & & 25.0 & & & & \\
\hline
\end{tabular}

\subsubsection{Species Similarity}

In analysing the similarities between species and years Figure represents a dendrogram showing similarities between the three different logged compartments. The shortest Euclidean distance of (Figure 5) 123.44 between year 2013 and 2017 (Table 11) show that they both have many plants species that are similar. The longest Euclidean distance of 179.11 between 2015 and 2107 depicts they both have less similar plants species. 


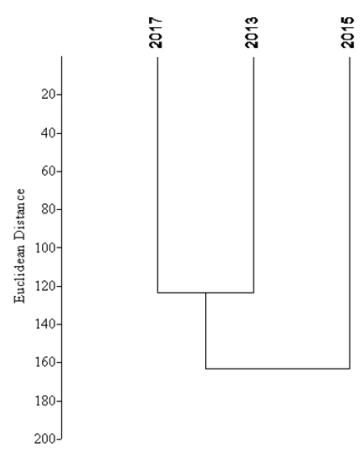

Figure 5. Euclidean Distance

Table 11. Similarities and distances

\begin{tabular}{llll}
\hline Year & $\mathbf{2 0 1 3}$ & $\mathbf{2 0 1 5}$ & $\mathbf{2 0 1 7}$ \\
\hline $\mathbf{2 0 1 3}$ & 0 & 147.26 & 123.44 \\
$\mathbf{2 0 1 5}$ & 147.26 & 0 & 179.11 \\
$\mathbf{2 0 1 7}$ & 123.44 & 179.11 & 0 \\
\hline
\end{tabular}

The structure of the Akak forest area is J reverse showing that the forest is growing to climax with (Figure 6) many individuals as recruits (2856 individuals).

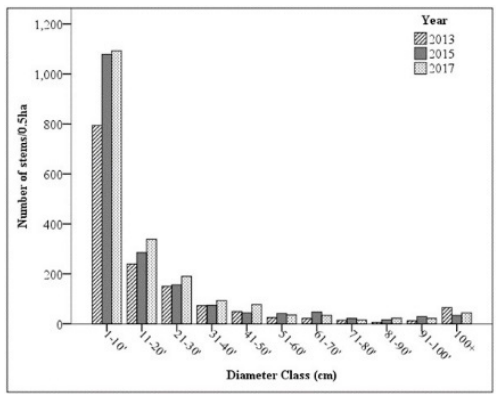

Figure 6. Structure of Akak forest area

\subsection{Regeneration Potential in Akak Forest Area}

Table 12. The status of regeneration potentials in Akak forest $(+)$ means presence and $(-)$ means absence

\begin{tabular}{|c|c|c|c|c|}
\hline Family & Scientific name & 2013 & 2015 & 2017 \\
\hline Acanthaceae & Justicia sp.1 & - & + & - \\
\hline \multirow{10}{*}{ Anacardiaceae } & Antrocaryon sp.1 & + & + & - \\
\hline & Lannea welwitschii & + & + & - \\
\hline & Sorindeia macrophylla & + & + & + \\
\hline & Sorindeia sp.1 & + & + & - \\
\hline & Sorindeia sp.3 & + & + & - \\
\hline & Sorindeia sp.4 & + & + & - \\
\hline & Sorindeia sp.5 & - & + & - \\
\hline & Sorindeia sp.6 & - & + & - \\
\hline & Sorindeia sp.7 & - & + & - \\
\hline & Sorindeia thompsonii & - & + & - \\
\hline \multirow{2}{*}{ Annonaceae } & Annickia chlorantha & - & & + \\
\hline & Anona sp.1 & - & + & - \\
\hline
\end{tabular}




\begin{tabular}{|c|c|c|c|c|}
\hline & Anonidium mannii & - & + & - \\
\hline & Anonidium sp.1 & + & + & - \\
\hline & Cleistopholis glauca & + & + & + \\
\hline & Cleistopholis patens & + & + & + \\
\hline & Cleistopholis sp.1 & - & + & + \\
\hline & Greenwayodendron sp.1 & + & + & + \\
\hline & Monodora myristica & - & - & + \\
\hline & Pachypodianthium staudtii & + & + & - \\
\hline & Pachypodianthium sp.1 & & + & - \\
\hline & Polyanthia suaveolens & + & + & - \\
\hline & Uvaria sp.1 & + & + & - \\
\hline & Uvariodendron sp.1 & + & - & + \\
\hline & Uvariopsis sp & - & - & + \\
\hline & Xylopia aethiopica & + & + & + \\
\hline & Xylopia quintasii & + & + & + \\
\hline \multirow{11}{*}{ Apocynaceae } & Xylopia staudtti & - & - & + \\
\hline & Alstonia boonei & + & - & - \\
\hline & Eglandulosa sp.1 & + & + & - \\
\hline & Holarrhena floribunda & + & - & - \\
\hline & Picralima nitida & + & - & + \\
\hline & Pleiocarpa sp.1 & + & + & + \\
\hline & Rauvolfia vomitoria & + & + & + \\
\hline & Tabernaemontana crassa & + & + & + \\
\hline & Tabernaemontana sp.1 & + & + & - \\
\hline & Voacanga sp.1 & - & - & + \\
\hline & Voacanga sp.2 & - & + & - \\
\hline \multirow{5}{*}{ Araceae } & Raphidophora sp.1 & + & - & + \\
\hline & Rektophylum mirabilis & + & + & - \\
\hline & Rektophylum sp.1 & + & - & - \\
\hline & Raphidophora sp.2 & - & + & - \\
\hline & Recktophylum sp.2 & - & + & - \\
\hline \multirow{7}{*}{ Arecaceae } & Elaeis guineensis & + & + & - \\
\hline & Elaeis sp.1 & - & + & - \\
\hline & Elaeis sp.2 & - & + & - \\
\hline & Raphia sp.1 & - & + & - \\
\hline & Calamus sp.1 & + & - & - \\
\hline & Calamus sp.2 & + & - & - \\
\hline & Calamus sp.3 & + & - & - \\
\hline Asparagaceae & Anthericum sp.1 & + & + & + \\
\hline Asteraceae & Chromolaena odorata & + & - & - \\
\hline \multirow{3}{*}{ Begoniaceae } & Begonia meyeri-johannis & - & + & - \\
\hline & Newbouldia laevis & + & - & + \\
\hline & Spathodia campanulata & - & - & + \\
\hline \multirow{6}{*}{ Burseraceae } & Canarium schweinfurthii & + & + & + \\
\hline & Dacryodes edulis & + & + & - \\
\hline & Dacryodes klaineana & - & + & - \\
\hline & Pseudospondias sp.1 & + & + & + \\
\hline & Santiria tremari & + & + & - \\
\hline & Santiria sp.1 & + & - & - \\
\hline \multirow{4}{*}{ Cecropiaceae } & Musanga cecropioides & + & + & - \\
\hline & Musanga sp.1 & - & + & - \\
\hline & Myrianthus arboreus & - & + & - \\
\hline & Myrianthus sp.1 & + & + & - \\
\hline \multirow{3}{*}{ Celastraceae } & Salacia sp.1 & + & + & + \\
\hline & Salacia sp.2 & + & + & - \\
\hline & Salacia sp.3 & - & + & - \\
\hline
\end{tabular}


Clusiaceae

Combretaceae

Commelinaceae

Connaraceae

Dichapetalaceae

Dilleniaceae

Ebenaceae

Euphorbiaceae

a.Fabaceae
Salacia sp.4

Salacia sp. 5

Allanblackia sp.1

Garcinia mannii

Garcinia ovalifolia

Garcinia sp.1

Garcinia sp.2

Garcinia sp.4

Terminalia ivorensis

Commelina sp.1

Palisota sp.1

Rourea sp.3

Dichapetalum angolense

Dichapetalum sp. 1

Dichapetalum sp.2

Dichapetalum sp.3

Dichapetalum sp.4

Dichapetalum sp.5

Tapura africana

Tetracera podotricha

Diospyros sp.4

Diospyros crassiflora

Diospyros hirsuta

Diospyros hoyleana

Diospyros mouloundouana

Diospyros sp.1

Diospyros hirsuta

Diospyros sp. 2

Diospyros sp.3

Diospyros sp.6

Diospyros suaveolens

Diospyros zenkeri

Alchornea sp. 1

Discoglypremna caloneura

Discoglypremna sp.1

Klaineanthus sp.1

Macaranga sp.1

Macaranga monandra

Macaranga sp.2

Macaranga sp.3

Maesobotrya sp.1

Manniophyton fulvum

Mareyopsis sp.1

Plagiostyles africana

Ricinodendron heudelotii

Ricinodendron heudelotii

Sapium ellipticum

Tetracarpidium sp.1

Angylocalyx pynaertii

Afzelia sp. 1

Afzelia sp. 2

Albizia adianthifolia

Albizia sp.1

Amphimax pterocarpoides

Anthonotha fragrans

Anthonotha macrophylla

\begin{tabular}{|c|c|c|}
\hline- & + & - \\
\hline- & + & - \\
\hline+ & - & - \\
\hline+ & + & - \\
\hline+ & - & - \\
\hline+ & + & + \\
\hline - & + & - \\
\hline+ & + & - \\
\hline+ & - & - \\
\hline+ & + & - \\
\hline+ & + & - \\
\hline- & + & + \\
\hline+ & + & + \\
\hline+ & + & - \\
\hline+ & + & - \\
\hline+ & + & - \\
\hline \multirow[t]{2}{*}{-} & + & - \\
\hline & + & - \\
\hline+ & + & + \\
\hline- & - & + \\
\hline+ & + & - \\
\hline+ & + & + \\
\hline+ & + & - \\
\hline+ & + & - \\
\hline+ & + & + \\
\hline- & + & - \\
\hline+ & + & + \\
\hline- & + & - \\
\hline \multirow[t]{2}{*}{+} & + & + \\
\hline & + & \\
\hline+ & + & + \\
\hline+ & + & - \\
\hline+ & - & - \\
\hline- & + & - \\
\hline- & + & + \\
\hline+ & + & - \\
\hline+ & + & - \\
\hline+ & + & - \\
\hline+ & + & - \\
\hline- & + & - \\
\hline+ & + & - \\
\hline+ & + & - \\
\hline- & + & - \\
\hline+ & + & - \\
\hline+ & + & + \\
\hline- & + & - \\
\hline - & - & + \\
\hline- & - & + \\
\hline+ & + & + \\
\hline+ & + & + \\
\hline+ & + & + \\
\hline+ & + & + \\
\hline- & + & - \\
\hline- & - & + \\
\hline- & + & - \\
\hline+ & + & + \\
\hline
\end{tabular}




\begin{tabular}{|c|c|c|c|c|}
\hline & Baphiopsis sp.1 & + & - & - \\
\hline & Baphia nitida & + & + & + \\
\hline & Brachystegia sp.2 & + & - & - \\
\hline & Calpocalyx sp.1 & + & + & + \\
\hline & Calpocalyx sp.2 & + & + & - \\
\hline & Calpocalyx sp.3 & + & + & - \\
\hline & Cylicodiscus gabunensis & + & + & - \\
\hline & Cynometra hankei & + & + & - \\
\hline & Dialium bipindense & + & + & - \\
\hline & Dialium guineense & + & - & - \\
\hline & Dialium lopense & + & + & - \\
\hline & Distemonanthus benthamianus & + & + & - \\
\hline & Detarium microcarpum & - & + & - \\
\hline & Erythrophleum guineense & - & + & - \\
\hline & Guibourtia sp.1 & + & + & + \\
\hline & Hylodendron gabunense & + & + & - \\
\hline & Millettia sp.1 & + & + & + \\
\hline & Monopetalanthus sp.1 & - & + & - \\
\hline & Millettia sp.2 & - & + & - \\
\hline & Parkia bicolor & - & + & - \\
\hline & Pentaclethra macrophylla & + & + & + \\
\hline & Piptadeniastrum africana & + & + & + \\
\hline & Pteropcarpus soyau+ii & + & + & - \\
\hline & Tetrapleura tetraptera & - & + & - \\
\hline Flacourtiaceae & Scotellia coriacea & - & - & + \\
\hline \multirow{2}{*}{ Gentianaceae } & Anthocleista nobilis & - & + & - \\
\hline & Anthocleista sp.1 & + & + & - \\
\hline Gnetaceae & Gnetum africanum & + & + & - \\
\hline Huaceae & Afrostyrax lepidophyllus & - & - & + \\
\hline \multirow{2}{*}{ Icacinaceae } & Lasianthera africana & + & + & - \\
\hline & Lavigeria sp.1 & + & + & + \\
\hline \multirow{4}{*}{ Irvingiaceae } & Desbordesia sp.1 & - & + & - \\
\hline & Desbordesia glaucescens & + & - & + \\
\hline & Irvingia gabonensis & + & + & - \\
\hline & Vitex grandifolia & - & + & - \\
\hline \multirow{3}{*}{ Lamiaceae } & Vitex micrantha & - & + & + \\
\hline & Vitex sp.1 & - & + & - \\
\hline & Vitex sp.2 & - & + & + \\
\hline \multirow{5}{*}{ Lauraceae } & Beilschmiedia sp.1 & - & - & + \\
\hline & Homalium letestui & - & + & + \\
\hline & Homalium sp.1 & + & + & - \\
\hline & Hypodaphnis zenkeri & + & + & - \\
\hline & Napoleonaea sp.1 & + & + & - \\
\hline \multirow{3}{*}{ Lecythidaceae } & Napoleonaea sp.2 & + & + & + \\
\hline & Napoleonaea sp.3 & - & + & - \\
\hline & Petersianthus sp.1 & - & + & + \\
\hline Leguminosae & Cylicodiscus gabunensis & + & - & - \\
\hline Leptaulaceae & Leptaulus sp.1 & - & - & + \\
\hline \multirow{8}{*}{ Malvaceae } & Ceiba pentandra & + & + & - \\
\hline & Christiana sp.1 & - & - & + \\
\hline & Cola accuminata & - & + & + \\
\hline & Cola cauliflora & + & + & + \\
\hline & Cola digitata & - & + & + \\
\hline & Cola lateritia & + & + & + \\
\hline & Cola millenii & - & + & + \\
\hline & Cola rostrata & + & + & + \\
\hline
\end{tabular}


Marantaceae

Melastomataceae

Meliaceae

Memecylaceae

Menispermaceae

Monimiaceae

Moraceae

Myristicaceae

Myrtaceae

Ochnaceae

Octoknemaceae
Cola sp. 1

Cola sp. 2

Duboscia macrocarpa

Grewia coriacea

Leptonychia sp.1

Leptonychia sp.2

Leptonychia sp.3

Scaphopetalum sp.1

Scaphopetalum sp.2

Sterculia tragacantha

Theobroma cacao

Sterculia tragacantha

Morantochloa sp.1

Dissotis sp. 1

Memecylon warnekii

Memecylon sp. 1

Memecylon sp.2

Carapa procera

Carapa sp. 1

Entandrophragma candollei

Entandrophragma sp.1

Guarea cedrata

Guarea sp.1

Guarea sp.2

Khaya sp.1

Trichilia rubescens

Trichilia sp. 1

Trichilia welwitschii

Trichoscypha abut

Trichoscypha acuminata

Turreanthus sp.1

Mamecylum sp.1

Stephania sp.1

Glossocalyx sp.1

Chlorophora excelsa

Dorstenia sp. 1

Ficus elasticum

Ficus mucuso

Ficus sp. 1

Ficus subsargitata

Ficus sur

Treculia africana

Treculia obovoidea

Treculia sp. 1

Trilepisium sp.1

Trilepisium madagascariense

Coelocaryon sp.1

Pycnanthus angolensis

Staudtia kamerunensis

Eugenia sp.1

Syzygium guineense

Syzygium sp. 1

Campylospermum sp. 1

Lophira alata

Ochna sp.1

Octoknema sp.1

\begin{tabular}{|c|c|c|}
\hline- & + & + \\
\hline+ & + & + \\
\hline+ & + & - \\
\hline+ & & + \\
\hline- & + & - \\
\hline- & + & + \\
\hline- & + & - \\
\hline+ & + & + \\
\hline+ & + & \\
\hline- & + & + \\
\hline+ & - & - \\
\hline - & + & - \\
\hline- & + & + \\
\hline- & + & - \\
\hline- & + & - \\
\hline- & + & - \\
\hline- & + & - \\
\hline+ & - & - \\
\hline- & + & + \\
\hline+ & - & - \\
\hline+ & + & - \\
\hline+ & + & - \\
\hline+ & + & + \\
\hline+ & + & - \\
\hline- & + & - \\
\hline+ & + & - \\
\hline+ & + & - \\
\hline+ & - & - \\
\hline- & + & - \\
\hline+ & + & - \\
\hline+ & + & - \\
\hline- & + & - \\
\hline- & + & + \\
\hline+ & + & - \\
\hline+ & + & - \\
\hline+ & + & + \\
\hline+ & + & - \\
\hline- & + & - \\
\hline- & + & - \\
\hline- & + & - \\
\hline- & + & - \\
\hline- & - & - \\
\hline+ & + & + \\
\hline- & + & - \\
\hline- & - & + \\
\hline+ & - & - \\
\hline+ & - & + \\
\hline+ & + & + \\
\hline+ & + & + \\
\hline- & + & - \\
\hline+ & - & + \\
\hline+ & - & - \\
\hline+ & + & + \\
\hline+ & + & - \\
\hline+ & - & - \\
\hline+ & - & + \\
\hline
\end{tabular}




\begin{tabular}{|c|c|c|c|c|}
\hline \multirow{6}{*}{ Olacaceae } & Diogoa zenkeri & + & - & - \\
\hline & Olax sp.1 & + & + & + \\
\hline & Strombosia grandifolia & + & + & - \\
\hline & Strombosia pustulata & + & + & - \\
\hline & Strombosia zenkeri & - & + & - \\
\hline & Strombosiopsis tetrandra & + & + & + \\
\hline Pandaceae & Microdesmis sp.1 & - & + & + \\
\hline \multirow[t]{3}{*}{ Passifloraceae } & Barteria fistulosa & - & + & - \\
\hline & Antidesma sp.1 & - & + & + \\
\hline & Bridelia sp.1 & - & - & + \\
\hline \multirow[t]{3}{*}{ Phyllanthaceae } & Magaritaria sp.1 & - & + & \\
\hline & Uapaca guineensis & - & + & + \\
\hline & Uapaca staudtii & - & - & + \\
\hline \multirow[t]{6}{*}{ Polygalaceae } & Carpolobia sp.1 & + & + & - \\
\hline & Drypetes molunduana & + & + & - \\
\hline & Drypetes sp.1 & + & + & + \\
\hline & Drypetes sp. 2 & - & + & - \\
\hline & Drypetes sp.3 & - & + & + \\
\hline & Drypetes sp.4 & + & + & + \\
\hline \multirow{7}{*}{ Putranjivaceae } & Drypetes sp. 5 & + & + & - \\
\hline & Drypetes sp.6 & - & + & + \\
\hline & Drypetes sp.7 & + & + & - \\
\hline & Drypetes sp. 8 & + & + & + \\
\hline & Drypetes sp.9 & + & + & - \\
\hline & Drypetes sp.10 & - & + & - \\
\hline & Drypetes sp.11 & - & + & - \\
\hline Rhizophoraceae & Anopyxis kleneanna & - & - & + \\
\hline \multirow[t]{11}{*}{ Rosaceae } & Pyracantha sp. 1 & + & + & - \\
\hline & Belonophora sp.1 & - & + & + \\
\hline & Belonophora sp.2 & - & + & - \\
\hline & Canthium sp.1 & + & - & + \\
\hline & Shumanniophyton magnificum & + & - & + \\
\hline & Corynanthe sp.1 & - & + & + \\
\hline & Ixora sp.1 & - & + & + \\
\hline & Massularia acuminata & + & + & - \\
\hline & Massularia sp.1 & + & - & - \\
\hline & Morinda lucida & - & + & - \\
\hline & Mitragyna sp & - & - & + \\
\hline \multirow[t]{11}{*}{ Rubiaceae } & Oxyanthus sp.1 & - & + & - \\
\hline & Pauridiantha sp.1 & + & + & - \\
\hline & Pavetta sp.1 & - & + & - \\
\hline & Rothmania hirpida & + & + & + \\
\hline & Rothmania sp.1 & + & + & - \\
\hline & Rothmania sp.2 & + & + & - \\
\hline & Rothmannia talbotii & + & + & + \\
\hline & Rothmania sp.3 & - & + & - \\
\hline & Rothmania sp.4 & - & + & - \\
\hline & Shebournia sp.1 & - & + & + \\
\hline & Tricalysia sp.1 & + & - & + \\
\hline \multirow{3}{*}{ Ruscaceae } & Dracaena arborea & + & + & - \\
\hline & Dracaena sp.1 & + & + & - \\
\hline & Fagara sp.1 & + & - & - \\
\hline \multirow[t]{2}{*}{ Rutaceae } & Zanthoxylum sp.1 & + & + & + \\
\hline & Zanthoxylum sp.2 & + & & + \\
\hline Salicaceae & Phyllobotryon sp.1 & - & + & - \\
\hline Sapindaceae & Blighia sp.1 & + & + & - \\
\hline
\end{tabular}




\begin{tabular}{|c|c|c|c|c|}
\hline & Chytranthus sp.1 & + & + & + \\
\hline & Chytranthus talbotii & - & + & + \\
\hline \multirow{5}{*}{ Sapotaceae } & Laccodiscus sp.1 & - & - & + \\
\hline & Baillonella toxisperma & - & - & + \\
\hline & Gambeya africanum & + & + & + \\
\hline & Gambeya sp.2 & + & - & - \\
\hline & Omphalocarpum sp.1 & + & - & - \\
\hline \multirow[t]{2}{*}{ Tiliaceae } & Desplatsia dewevrei & + & + & - \\
\hline & Celtis sp.1 & + & + & + \\
\hline \multirow[t]{4}{*}{ Ulmaceae } & Celtis sp.2 & + & + & - \\
\hline & Trema guineensis & - & + & + \\
\hline & Rinorea dentata & + & + & + \\
\hline & Rinorea oblongifolia & + & - & + \\
\hline \multirow{4}{*}{ Violaceae } & Rinorea sp.1 & + & + & - \\
\hline & Rinorea sp.2 & + & + & - \\
\hline & Rinorea sp.3 & + & + & - \\
\hline & Rinorea sp.4 & - & + & - \\
\hline Vitaceae & Cissus dinklagei & + & - & - \\
\hline \multirow{2}{*}{ Zingiberaceae } & Hydrychium sp.1 & - & + & - \\
\hline & Renealmia sp.1 & - & + & - \\
\hline
\end{tabular}

Result show that the family Fabaceae species have the highest presence of regeneration regenerating species (31) followed by the family Rubaceae with (21) species (Table 12).

Most of the regenerating species in Akak forest are between the size class 1-4.9 (Figure 7) with the year 2015 having the highest number of recruits (1014 individuals) (Table 12).

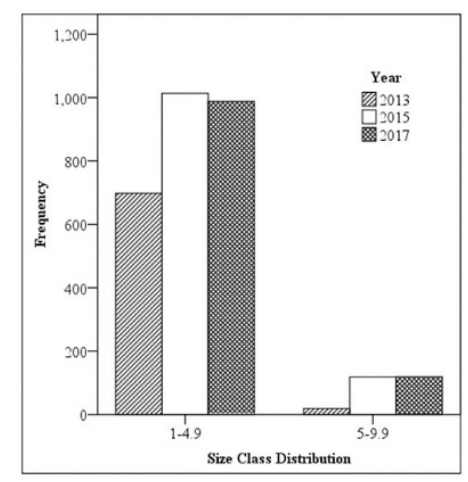

Figure 7. Regeneration potentials

\section{Discussion}

\subsection{Floristic Composition of Akak Forest Area}

Tropical forest ecosystems are one of the richest terrestrial ecosystems which support a variety of life forms and maintain huge global biodiversity (Shi \& Singh, 2002). The forests of southwestern Cameroon are generally known to be rich in species diversity because they are located within the high rainfall zone of the Guinean equatorial tropical forest. (Fonge et al., 2013). Numbers of species, genera, families, were higher in the in 2015 logged compartments compared to the 2017 and 2013 this can be due to the increase in densities of seedlings. These findings are consistent with the general notion that the recovery of forests after logging normally takes years and the resulting forest differ considerably in species composition, and structure from the original forest (Johns, Barreto, \& Uhl, 1996; Kinjanjui, Karachi, \& Kennedy, 2013; Wiafe, 2014). Felton, Felton, Wood and Lindenmayer (2006) found significantly higher proportion of regenerated pioneers in logged sites in a Bolivian subtropical forest. Following disturbances, pioneers and Non Pioneer Light demanding densities are expected to increase whilst those of shade-bearers decline with time until the canopy closes (Duah-Gyamfi, Kyereh, Adam, Agyeman, \& Swaine 2012). The higher numbers of species, genera, families in 2015 logged compartments compared to 2017 and 2013 can be attributed to the openings of canopy gaps and higher incidence of sunlight on the forest floors as a result of 
logging disturbance and the time for pioneers to colonise the environment (Hawthorne, 1993; Swaine, Agyeman, \& Adam, 1998; Molino \& Sabatier, 2001). This result is also in line with the observations by Tchouto et al. (2004) in Cameroonian forest that herbaceous species, pioneer species and climbers increase with the degree of disturbances. Similarly, the relatively higher numbers of species, genera, families recorded in compartment logged 3 years (2015) ago compared to compartment logged 1 year (2017) suggest that more time may be required for the expected compositional of shade tolerant seedlings. The slight decrease in numbers of species, genera, families in 2013 as compared to 2015 is similar to Duah-Gyamfi et al. (2012) reported a reduction in the proportion of understory pioneer species after 33 months of selective logging in Pra Anum Forest Reserve of Ghana. The greater basal area of species in 2017 compared to 2015 may be due immediately after logging there is still a grater number of plants stands but will eventually change with time since the mortality rate of plants adapted to low light levels will eventually increase. Other investigators have reported higher basal areas following disturbances in different forests (Latty, Canham, \& Marks, 2004; Sefah, unpublished). Sefah (unpublished), for example, found significantly higher mean basal area of seedlings and saplings in heavily disturbed forests forest types in Ghana, and concluded that this might be due to the sufficient light availability resulting from the low canopy cover.

\subsection{Overall Importance of Plant Species in Akak Forest Area}

In the study area, the most dominant family was the Fabaceae (99) species this is in line with Sainge (2016) who reported a similar situation in the kimbi fundong park. Fabaceae always fall among the three most dominant families in the world. Fabaceae is the most diverse plant family in the world with a wide distribution of sort, registering 770 genera and 19,500 sorts, and considered the third largest family of angiosperms in species numbers (Beech, Rivers, Oldfield, \& Mith, 2017; Azani et al., 2017) the second most dominant family is Rubiaceae, with 91 species. This is inline with (Ndam, Nkefor, \& Blackmore, 2001) and Fonge et al. (2011) who reported that the Rubiaceae was the most dominant tree family in the Mount Cameroon region and Kenfack et al. (2007) also report Rubiaceae to be the most dominant tree family in the Korup National Park. Nevertheless, Rubaiaceae could be the most dominant tree family in the Guinean equatorial forest as reported by Fonge et al. (2013).

It is an established fact that few species dominate forest ecosystems that experience prolonged absence or frequent occurrences of high intensity disturbances (Cornell, 1978; Sheil \& Burslem, 2003; Kinjanjui, Karachi, \& Kennedy, 2013). This pattern was evident in the results of the present study, which showed a much higher importance value for the fifteen most dominant species in the 2013 logged compartment as compared to compartment logged in 2017 and 2013. Similar results have been observed in the tropics (Todaria, Uniyal, Pokhriyal, Dasgupta, \& Bhatt, 2010; Nartey, 2013 unpublished; Wiafe, 2014), though other studies have produced contradictory results (Muhanguzi, Obua, \& Oryem-Origa, 2007; Chazdon et al., 2010). With time, the importance of the dominant species diminished in the studied habitats this is evident in our study sites were logged sites of 2017 had higher IVIs for the fifteen most dominant species than sites logged in the year 2015.

Resistance is the inherent ability of a forest to remain unaffected or absorb moderate disturbances (Thompson, Mackey, Mcnulty, \& Mosseler, 2009). According to Belote, Jones, \& Weiboldt (2012), forest resistance decreases with increasing timber-harvesting disturbance when these researchers found that compositional stability was lower in most disturbed plots of forests in the Appalachian Mountains of North America. Thus, the dominance of similar species in the 2013, 2015 and 2017 sites by the same families in the present study reflects high resistance to logging disturbance, coupled with their relatively high diversity. According to Thompson et al. (2009), resistance is enhanced by increase diversity of a forest ecosystem. The dominance of Angylocalyx pynaertii, Staudtia kamerunensis, Cola millenii, Drypetes molunduana, Tapura Africana, Baphia nitida, Sorindeia macrophylla and Musanga cecropioides in at least two sites in terms of importance in most species are either pioneers or nonpioneer light demanding species (Duah-Gyamfi, Kyereh, Adam, Agyeman, \& Swaine, 2012).

\subsection{Ecological Structure of the Akak Forest Area}

Generally, the Akak forest area has a high diversity. The higher species diversity in the 2017 as opposed 2013 and 2015 This is in agreement with Young and Swiacki (2006) who stated that diversity was made up of the variety of species present and the relative abundance of those species. The higher the values, the higher the diversity (Ojo, 2004). The Simpson's Index indicates that the diversity of the reserve is high and it is an indicative of a healthy reserve when compared to the other years. The specie richness index obtained was higher in 2015 (137.7) than in the other years which indicate high species richness this contradicts Fonge et al. (2013) who had a poor specie richness in her studies on Plants in Lewoh-Lebang in the Lebialem Highlands of Southwestern Cameroon.

\subsection{Regeneration Potential in Akak Forest Area}

Recruitment of viable seedling and saplings can be indicators of the regeneration status of a plant community. The processes involved in tree regeneration can be influenced by disturbance regimes and other factors, such as 
predation, canopy openness, soil moisture availability, as well as biological features of the species, such as their life cycles and behavior. The absence of some regeneration species in some years such as Lophira alata in the year 2017, Terminalia ivorensis in the year 2015 and Tetrapleura tetraptera in 2017 can be attributed excessive selective logging of this species for commercial purposes. other factors, such as environmental gradient, as well as species-specific regeneration patterns together with disturbance regimes contributed to the non presence of these species. The presence of abundant seedlings of the other species (Afrostyrax lepidophyllus, Pentaclethra macrophylla, Ricinodendron heudelotii and Scorodophloeus zenkeri) is consistent with a previous report from tropical rainforests (Whitmore, 1996). their characterized by sufficient numbers of seedlings, saplings and adults, indicate that they are regenerating successfully. for Afrostyrax lepidophyllus and Pentaclethra macrophylla, their superior natural regeneration can be linked to their good fruiting efficiency and germination capacity. in addition, the high fruit predation of Afrostyrax lepidophyllus by small mammals ensures the dissemination of seeds throughout the forest. Pentaclethra macrophylla has fruits that explode at maturity, scattering seeds at great distances from seed tree, which reduces the density-dependent mortality that occurs when too many seeds germinate at the same time under a seed tree. Ricinodendron heudelotii, is a gap opportunist and fast-growing pioneer tree (Plenderleith, 2000) that regenerates rapidly in open canopy forest and very poorly under closed canopies. its high regeneration observed in this study can be attributed to the high degree of disturbances in the forest.

\section{Conclusion}

The Akak forest area important in terms of plant biodiversity with 71 families and 384 species recorded in the study area and also considered as important destination point for rich timber resources such as Diospyros crassiflora, Petersianthus macrocarpus, Entandrophragma cylindricum, Khaya ivorensis, Piptadeniastrum africanum, Milicia excelsa, Lophira alata, Strombosia pustulata etc are found. Hence, it is very crucial to protect these important forests for biodiversity, sustainable management and environmental perspectives. The forest has a high diversity and richness with Angylocalyx pynaertii, Staudtia kamerunensis, Cola millenii, Drypetes molunduana, Tapura Africana, Baphia nitida, Sorindeia macrophylla and Musanga cecropioides figuring out as dominant species found Akak forest area. The Akak forest area currently displays signs of recovery. Evidently, the forest area is very rich in trees in the lower diameter classes, which is an indication of a healthy and vigorous stand. The structure of the forest tells us that there is high species diversity and the relative richness in plant species of the and it is in the process of recovering.

\section{Conflict of interests}

The authors declare that there is no conflict of interests regarding the publication of this paper.

\section{References}

Akinsanmi, F. A. (1999). Updating Environmental Information in Nigeria (p. 15). Invited Lead paper presented at the 26th Annual Conference of the Forestry Association of Nigeria held in Maiduguri, Borno.

Azani, N., Babineau, M., Bailey, C. D., Banks, H., Barbosa, A. R., Pinto, R. B., Boatwright, J. S., Borges, L. M., Brown, G. K., Bruneau, A., \& Candido, E. (2017). A new subfamily classification of the Leguminosae based on a taxonomically comprehensive phylogeny The Legume Phylogeny Working Group (LPWG). Taxon, 66(1), 44-77.

Barnes, B., Zak, D., Denton, S., \& Spurr, S. (1998). Forest Ecology (4th ed.). New York, NY Wiley.

Beech, E., Rivers, M., Oldfield, S., \& Mith, P. P. (2017). Global tree search: The first complete global database of tree species and country distributions. Journal of Sustainable Forestry, 36(5), 454-489.

Beentje, H. J. (1996). Centres of Plant Diversities in Africa, the Biodiversity of African Plants. Kluwer Academic Publishers, TheNetherlands.

Belote, R. T., Jones, R. H., \& Weiboldt, T. F. (2012). Compositional Stability and Diversity of Vascular Plant Communities following Logging Disturbance in Appalanchian Forests. EcoAppl., 22(2), 502-516.

Bhuyan, P., Khan, M. L., \& Tripathi, R. S. (2003). Tree diversity and population structure in undis-turbed and human-impacted stands of tropical wet evergreen forest in Arunachal Pradesh, eastern himalayas, India. Biodiversity and Conservation, 12(8), 1753-1773.

Ceccon, E., Huante, P., \& Rincón, E. (2006). Abiotic factors influencing tropical dry forests regeneration. Brazilian Archives of Biology and Technology, 49(2), 305-312. 
Chazdon, R. L., Finegan, B., Capers, R. S., Salgado-Negret, B., Casanoves, F., Boukili, V., \& Norden, N. (2010). Composition and Dynamics of Functional Groups of Trees During Tropical Forest Succession in Northeastern Costa Rica. Biotropica, 42(1), 31-40.

Cornell, J. H. (1978). Diversity in Tropical Rain Forests and Coral Reefs. Science, 199, 1302-1310.

Duah-Gyamfi, A., Kyereh, B., Adam, K. A., Agyeman, V. K., \& Swaine, M. D. (2012). Natural Regeneration Dynamics of Tree Seedlings on Skid Trails and Tree Gaps Following Selective Logging in a Tropical Moist Semi-Deciduous Forest in Ghana. Open Journal of Forestry, 4(1), 49-57.

Duchok, R., Kenyusen, K., Ashalata, D. K., Ashish, P., \& Khan, M. L. (2005). Population structure and regeneration status of medicinal tree Illicium griffithii in relation to disturbance gradients in temperate broadleaved forest of Arunachal Pradesh. Current Science, 89(4), 673-676.

FAO. (2010). Global Forest Resources Assessment 2010, Main report. FAO Forestry Paper, 163; FAO Rome, 340.

Felton, A., Felton, A. M., Wood, J., \& Lindenmayer, D. B. (2006). Vegetation Structure, Phenology, and Regeneration in the Natural and AnthropogenicTtree-fall Gaps of a Reduced-impact Logged Subtropical Bolivian Forest. Forest Ecology and Management, 235, 186-193.

Fonge, B. A., Focho, D. A., Egbe, E. A., Tening, A. S., Fongod, A. N., \& Neba, G. A. (2011). The effects of climate and edaphic factors on plant colonisation of lava flows on Mount Cameroon. Journal of Ecology and the Natural Environment, 3(8), 255-267.

Fonge, B. A., Tchetcha, D. J., \& Nkembi, L. (2013). Diversity, Distribution, \& Abundance of Plants in LewohLebang in the Lebialem Highlands of Southwestern Cameroon. International Journal of Biodiversity, 13.

Gebreselasse, G. V. (2011). Plant communities' species diversity seedling bank and re-sprouting in Nandi Forest, Kenya. PhD Thesis, Universitat Koblenz-Landau.

Gillespie, T. W., Brock, J., \& Wright, C. W. (2004). Prospects for quantifying structure, floristic composition and species richness of tropical forest. Int. J. Remote Sens., 25, 70-7.

Groenendijk, P. (2015). Long-term trends in tropical tree growth: A pantropical study [PhD thesis].

Hall, J. S., Harris, D. J., Medjibe, V., \& Ashton, P. M. S. (2003). The Effects of Selective Logging on Forest Structure and Tree Species Composition in a Central African Forest: Implications for Management of Conservation Areas. Forest Ecology and Management, 183, 249-264.

Hawthorne, W. D. (1993). Forest Regeneration after Logging: Findings of a Study in the Bia South Game Production Reserve Ghana. ODA Forestry Series No.3. Natural Resources Institute, Chatham Maritime, London.

Hossain, M., Rahman, L., Hoque, A., \& Khairul, M. (2004). Comparative regeneration status in a natural forest and enrichment plantations of Chittagong (south) forest division, Bangladesh. Journal of Forestry Research, $15,255-260$.

IUCN. (2010). Plants under pressure - a global assessment. The first report of the IUCN Sampled Red List Index for Plants. Royal Botanic Gardens, Kew, UK, Natural History Museum, London, and International Union for Conservation of Nature.

Johns, J. S., Barreto, P., \& Uhl, C. (1996). Logging Damage during Planned and Unplanned Logging Operations in the Eastern Amazon. Forest Ecology and Management, 89, 59-77.

Kenfack, D., Thomas, W., Chuyong, G., \& Condit, R. (2007). Rarity and abundance in a diverse African forest. Biodiversity and Conservation, 16(7), 2045-2074.

Kinjanjui, J. M., Karachi, M., \& Kennedy, K. N. (2013). Natural Regeneration and Ecological Recovery in Mau Forest Complex, Kenya. Open Journal of Ecology, 3(6), 417-422.

Latty, E. F., Canham, C. D., \& Marks, P. M. (2004). The Effects of Land-Use History on Soil Properties and Nutrient Dynamics in Northern Hardwood Forests of the Adirondack Mountains. Ecosystems, 7, $193-207$.

Mbolo, M. (2002). La collecte et l'analyse des donn'ees statistique sur les produits forestiers non ligneux: une 'etude pilote au Cameroun. D'epartement des forets. In Programme produit forestiers, non ligneux, FAO, Rome, Italie.

Mbwambo, L., Meilby, H., Ngaga, Y. M., Theilade, I., Treue, T., Van Beukering, P., Vyamana, V. G., \& Turner, R. K. (2011). The Importance of Local Forests Benefits: Valuation of Non-timber Forest Products in Eastern 
Arc Mountains in Tanzania, Working Paper 2011-05. Centre for Social and economic research on the global Environment, Norwich.

Molino, J. F., \& Sabatier, D. (2001). Tree Diversity in Tropical Rain Forests: A Validation of the Intermediate Disturbance Hypothesis. Science, 294, 1702-1704.

Muhanguzi, H. D. R., Obua, J., \& Oryem-Origa, H. (2007). The Effect of Human Disturbance on Tree Species Composition and Demographic Structure in Kalinzu Forest Reserve, Uganda. African Journal of Ecology, 45, $2-10$.

Nartey, A. O. (2013). Impact of Land Use Changes on Diversity, Community Structure and Natural Regeneration of Woody Plant Species in a Moist Semi-Deciduous Forest. A Thesis Submitted to the Department of Theoretical and Applied Biology in Partial Fulfillment of the Requirements of Master of Science Degree in Environmental Science, KNUST (Unpublished).

Nchanji, A. C., \& Plumptre, A. J. (2003). Seed germination and early seedling establishment of some elephantdispersed species in Banyang-Mbo Wildlife Sanctuary, south-western Cameroon. Journal of Tropical Ecology, 19, 229-237.

Ndam, N., Nkefor, J. P., \& Blackmore, P. (2001). Domestication of Gnetum africanum and G.buchholzianum (Gnetaceae), Overexploited wild forest vegetables of the Central African Region. Systematics and Geography of Plants, 71(2), 739-745.

Onyekwelu, J. C., Mosandl, R., \& Stimm, B. (2008). Tree species diversity and soil status of primary and degraded tropical rainforest ecosystems in South-Western Nigeria. Journal of Tropical Forest Science, 20(3), $193-204$.

Plenderleith, K. (2000). Ricinodendron heudelotii: A State of Knowledge Report Undertaken for the Central African Regional Program for the Environment. Oxford Forestry Institute/University of Oxford, Oxford.

Pritts, M. P., \& Hancock, J. E. (1983). The effect of population structure on growth patterns of the weedy goldenrod Solidago pauciflosculose. Canadian Journal Botany, 61, 1955-1958.

Saxena, A. K., \& Singh, J. S. (1984). Tree population structure of certain himalayan forest asso-ciations and implications concerning their future composition. Vegetatio, 58(2), 61-69.

Schaafsma, M., Morse, S. J., Posen, P., Swetnam, R. D., ..., \& Turner, R. K. (2011). The Importance of Local Forests Benefits: Valuation of Non-timber Forest Products in Eastern Arc Mountains in Tanzania, Working Paper 2011-05. Centre for Social and economic research on the global Environment, Norwich.

Schmitt, C. B., Burgess, N. D., Coad, L., Belokurov, A., ..., \& Winkel, G. (2009). Global analysis of the protection status of the world's forests. Biological Conservation, 142(10), 2122-2130.

Sefah, L. (2012). Community Diversity, Structure and Natural Regeneration of Trees in Tropical Forest: Effects of Human Disturbance. A Thesis Submitted to the Department of Environmental Science in Partial Fulfillment of the Requirement for the Award of the Master of Science (MSc.) Degree in Environmental Science, KNUST (Unpublished).

Sheil, D., \& Burslem, D. F. R. P. (2003). Disturbing Hypotheses in Tropical Forests. Trends in Ecology and Evolution, 18, 18-26.

Shi, H., \& Singh, A. (2002). An assessment of biodiversity hotspots using remote sensing and GIS. Journal of the Indian Society of Remote Sensing, 30, 105-112.

Silva, J. M. N., De Carvalho, J. O. P., De Lopes, J. C. A., De Almeida, B. F., Costa, D. H. M., De Oliveira, L. C., Vanclay, J. K., \& Skovsgaard, J. P. (1995). Growth and Yield of a Tropical Rain Forest in the Brazilian Amazon 13 Years after Logging. Forest Ecology and Management, 71, 267-274.

Stephanie, Y., \& Swiacki, L. N. (2006). Surveying the Forest Biodiversity of Evansburg State Park:Plant Community Classification and Species Diversity Assessment. International Journal of Botany.

Sunderland, T. C. H., Comiskey, J. A., Besong, S., Mboh, H., Fonwebon, J., \& Dione, M. A. (2003). Vegetation Assessment of Takamanda Forest Reserve, Cameroon. Smithsonian Institution.

Swaine, M. D., \& Whitmore, T. C. (1988). On The Definition of Ecological Species Groups in Tropical Rain Forests. Vegetation, 75, 81-86.

Swaine, M. D., Agyeman, V. K., \& Adam, A. R. (1998). Tree Regeneration after Logging in Two Forest Reserves in Ghana. Paper Presented At The International Conference on Value-Added Processing and Utilization of Lesser-Used Timber Species. Kumasi, Ghana. 
Tchouto, M. G. P. (2004). Plant diversity in a central African rainforest.Implications for biodiversity conservation in Cameroon. University of Edinburgh/Royal Botanic Garden of Edinburgh.

Tchouto, M. G. P., De Boer, W. F., De Wilde, J. J. F. E., Van Der Maesen, L. J. G., Yemefack, M., \& Cleef, A. M. (2004). Plant Diversity in a Central African Rain Forest, Implications for Biodiversity Conservation in Cameroon. Retrieved from http://www.Tropenbos.Org/ Country_Programmes/Cameroon/Publications? Page $=2$

Tesfaye, G., Teketay, D., \& Fetene, M. (2002). Regeneration of fourteen tree species in Harenna forest, southeastern Ethiopia. Flora-Morphology, Distribution. Functional Ecology of Plants, 197(6), 461-474.

Thompson, I., Mackey, B., Mcnulty, S., \& Mosseler, A. (2009). Forest Resilience, Biodiversity, and Climate Change. A Synthesis of the Biodiversity/Resilience/Stability Relationship in Forest Ecosystems. Secretariat of the Convention on Biological Diversity, Montreal. Technical Series, (43), 67.

Todaria, N. P., Uniyal, P., Pokhriyal, P., Dasgupta, S., \& Bhatt, D. (2010). Plant Diversity in Two Forest Types along the Disturbance Gradient in Dewalgarh Watershed and Garhwal Himalaya. India, Garhwal University.

Turner, I. M. (2001). The Ecology of Trees in the Tropical Rainforest.Cambridge University Press. Cambridge, 298.

UN. (1990). Retrieved from http://www.un.org/millenniumgoals

UN. (1992). Retrieved from http:/www.un.org/esa/sustdev/documents/ageda21

Whitmore, T. C. (1996). A review of some aspect of tropical rain forest seedlings ecology withsuggestions for further enquiry. In M. D. Swaine (Ed.), The Ecology of Tropical Forest Tree Seedlings (pp. 3-39). UNESCO, Paris.

Wiafe, E. D. (2014). Tree Regeneration after Logging in Rain-Forest Ecosystem. Research Journal of Biology, 2 , $18-28$.

\section{Copyrights}

Copyright for this article is retained by the author(s), with first publication rights granted to the journal.

This is an open-access article distributed under the terms and conditions of the Creative Commons Attribution license (http://creativecommons.org/licenses/by/4.0/). 